\title{
DYNAMICAL LIMITATIONS OF SINGLE-STAGE PMD COMPENSATORS
}

\author{
Ernesto Ciaramella ${ }^{1}$ \\ ${ }^{1}$ Scuola Superiore Sant'Anna, V. Cisanello 145 (56124) Pisa ITALY; \\ email:ernesto.ciaramella@cnit.it
}

Abstract: Dynamical effects in single-stage polarization-mode dispersion compensators are investigated. A typical compensator can actually work in two different operating modes. Dynamic evolution of the input signal can require a continuous switch between these modes, producing significant performance degradation. We introduce a numerical procedure to simulate dynamical effects, which confirms the occurrence of critical configurations.

\section{INTRODUCTION}

Polarization Mode Dispersion (PMD) is the main limiting effect for future $40 \mathrm{Gbit} / \mathrm{s}$ optical systems [1]. In a fiber link, PMD impairments depend on the State Of Polarization (SOP) of the input signal and on the fiber parameters (to first order approximation: Differential Group Delay, DGD, and Principal States of Polarization, PSPs). As they are statistically varying [2], the Outage Probability $(\mathrm{OP})$ concept [3] is used to evaluate system impairments.

The effectiveness of PMD-Compensators (PMDCs) is estimated by means of the Outage Probability (OP) as well [4]. To this aim, demanding numerical simulations are required, which first model the signal propagation, then optimise the PMDC (by setting it to an absolute or local maximum) and finally evaluate the penalty.

This quasi-static approach can be quite questionable. When we assume that the PMDC can be at a proper operating point at any time, we neglect what may happen during transients from one operating condition to the following. In 
principle, any change of the input SOP (State Of Polarisation) and/or of the fibre PMD corresponds to a time-dependent trajectory of the PMDC variable parameters.

Therefore the quasi-static assumption would be correct only if these trajectories had no discontinuity. If they have, no practical PMDC could have such a short response time to reach the next operating point in one or few bits; hence a PMD-compensated system could manifest unexpected degradation, with higher error rate (whatever good the OP could be).

Here we show how dynamical effects can arise in feedback-based PMDCs, with one fixed-delay stage.

\section{THEORETICAL MODEL}

We use a simplified first order model to explain the critical PMDC dynamics. Let us concentrate on IM-DD optical signals and also assume that the signal SOP is varying very fast, compared to the fibre PSP's (Principal States of Polarisation) and DGD (Differential Group Delay), which is a realistic case.

As known, PMDCs can typically use one of two different compensation strategies [1]. Up to $1^{\text {st }}$ order PMD approximation, system impairments only depend on $|\vec{\Omega} \times \vec{s}|$, where $\vec{s}$ and $\vec{\Omega}$ are the Stokes vectors of the signal SOP and overall $1^{\text {st }}$ order PMD, respectively ( $\vec{\Omega}$ is the vectorial combination of PMD of the fibre and of the PMDC, $\vec{\Omega}_{F}$ and $\vec{\Omega}_{C}$ respectively [4]). Hence a PMDC works effectively if either $\vec{\Omega}$ is parallel to $\vec{s}$ or if $|\vec{\Omega}|$ is simply minimised [4].

Let us now investigate one particular dynamical case, shown in Fig. 1. On the left, the signal is injected in one fibre PSP (i.e., $\vec{s}$ is parallel to

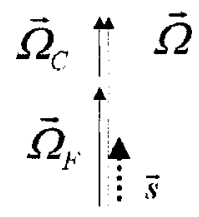

(a)

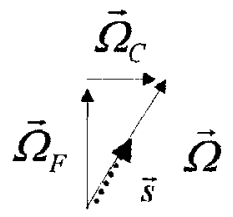

(b)

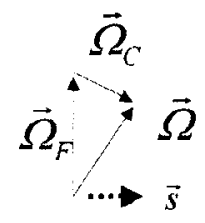

(c)

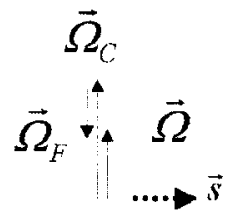

(d)

Figure 1 PMDC dynamics starting from input SOP in one PSP (a). As SOP changes (b, c), the PMDC should switch to the minimisation mode (d). 
$\left.\vec{\Omega}_{F}\right)$ and the PMDC aligns $\vec{\Omega}_{C}$ to both. However, this is a critical choice: if the signal SOP is rotating (b), it may reach an angle where this strategy cannot work anymore (c). In that configuration, the PMDC would suddenly have only one operating mode (shown in Fig. $1 \mathrm{~d}$ ) and it should switch to the minimization strategy. However this switching cannot be done instantaneously: hence during a limited time window, the PMDC is not opti-mised and may produce eye closure and error bursts. We outline that the usual OP treatment neglects this effect (and possible others, which are not discussed), as it would never show configuration (c).

As can be seen, if $\left|\vec{\Omega}_{F}\right|<\left|\vec{\Omega}_{C}\right|$ (i.e. $\mathrm{DGD}_{\mathrm{F}}<\mathrm{DGD}_{\mathrm{C}}$ ) a PMDC might be able to use the parallel mode for any SOP rotation. Yet this is an unpractical choice. As $\left|\vec{\Omega}_{F}\right|$ statistically varies, $\left|\vec{\Omega}_{C}\right|$ should be very large, but this can produce a nonnegligible $2^{\text {nd }}$ order PMD, when combined with $\vec{\Omega}_{F}$. This was already shown by the static analysis, as $\mathrm{DGD}_{\mathrm{C}}$ should be optimised to have the minimum OP, e.g., in a $40 \mathrm{~Gb} / \mathrm{s}$ system for one-section and $0.3<\left(<D G D>/ T_{b i t}\right)<0.5$ the optimum is $\mathrm{DGD}_{\mathrm{C}} \approx 16 \mathrm{ps}$ [4].

Using this optimum $\mathrm{DGD}_{\mathrm{C}}$ value, dynamical limitations might arise for $\left|\vec{\Omega}_{F}\right|$ greater than around $64 \%$ the bit time $T_{b i t}$ : this is, however, the DGD range where the PMDC beneficial effects would be really needed.

\section{NUMERICAL SIMULATIONS}

We introduce here for the first time a numerical technique to simulate PMDC dynamics. We condsider a $40 \mathrm{Gbit} / \mathrm{s}$ chirp-free NRZ signal. Using the wave-plate model [6], a 64-bit sequence is propagated in a fibre having 8 ps $\left(\approx \mathrm{T}_{\text {bit }} / 3\right)$ mean DGD. Among the various random wave-plate configurations, the software can select those having a chosen DGD. The PMDC has a one-stage with fixed delay $\left(\mathrm{DGD}_{\mathrm{C}}\right)$ and a Polarisation Controller $(\mathrm{PC})$, i.e. it basically has two Degrees of Freedom (DoF). The receiver is a fast photodiode followed by a Bessel filter ( $4^{\text {th }}$ order, $28 \mathrm{GHz}$ bandwidth). As the feedback signal, we use the eye opening. Note that, to simplify the analysis, no noise is added hence the eye opening is in principle the best possible feedback.

The dynamics is modelled as follows. First, we set an initial signal SOP and run an exhaustive search in all the PMDC configurations, optimising the two DoFs of the PMDC. After the starting configuration has been found, the signal SOP is varied by small steps, i.e., less than $0.02 \mathrm{rad} /$ iteration on both azimuth and ellipticity [7], and the PC is re-optimised. Now, to simulate a continuous evolution, in any iteration both PC angles start from the previous condition and cannot change by more than a fixed amount $(0.2 \mathrm{rad})$.

We first investigate the performance when $\mathrm{DGD}_{\mathrm{C}}=16 \mathrm{ps}$, i.e. the optimum 
value [5]. Obtained results show two situations. In a first (very common) case, the PMDC starts in a stable condition, the PC parameters do not change significantly for any SOP state and no significant penalty is observed. This suggests that if the PMDC starts in the minimisation mode, it locks firmly. However, in some cases the PMDC can start in an unstable condition, so that SOP rotations may produce severe eye closure (i.e., <-1.5 dB). After the eye closes, the PMDC may either recover to a stable condition or it may also reach another unstable condition (in which case, eye closure can occur again). Even allowing for the SOP to change at a slower rate, the same effects are obtained. As expected, these catastrophic events are likely obtained if $\mathrm{DGD}_{\mathrm{F}}$ is high and the input SOP is very close to one PSP when the PMDC starts.
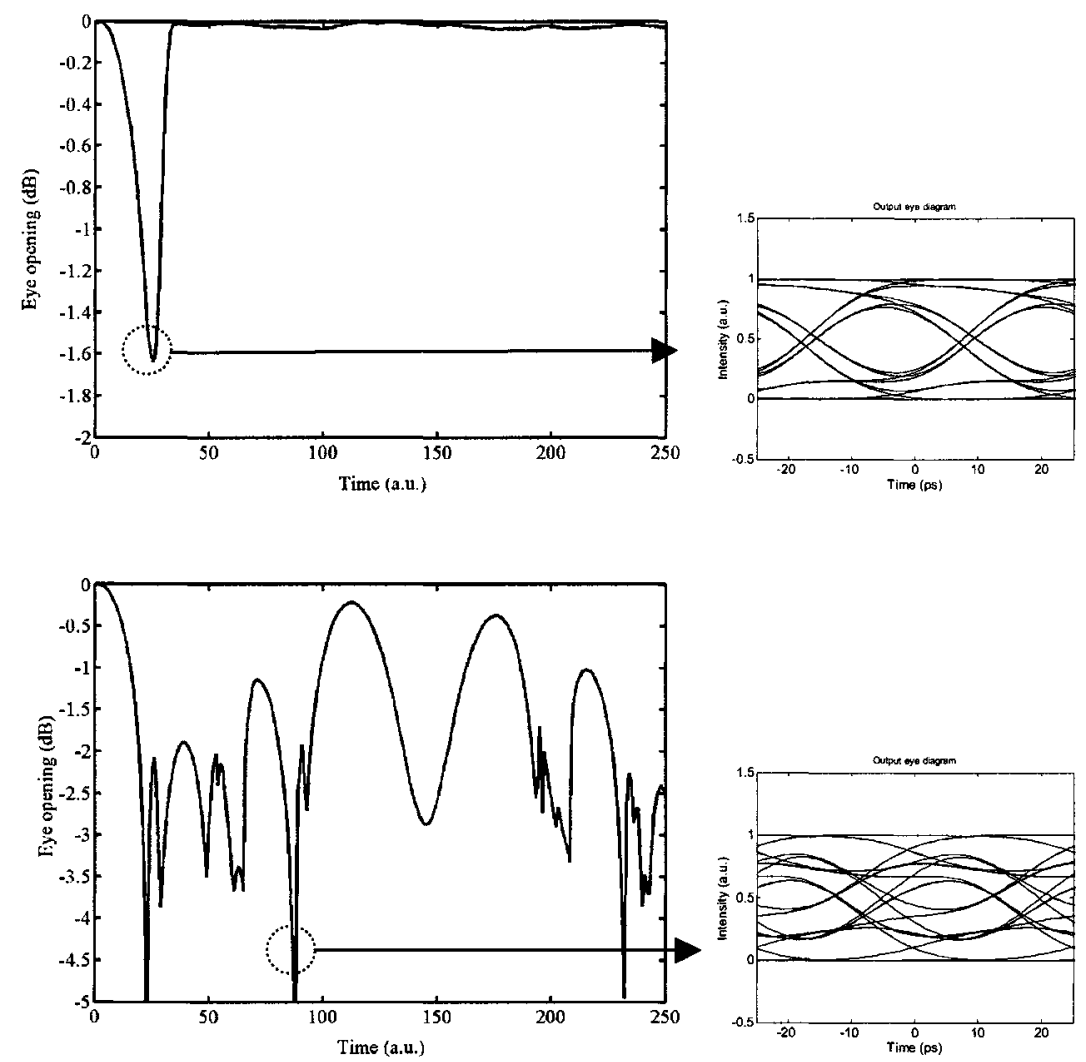

Figure 2 Eye closure evolution for a PMDC with $\mathrm{DGD}_{\mathrm{C}}=16 \mathrm{ps}$. We report the eye closure versus time for two fiber cases $\left(1^{\text {st }}\right.$ row: fibre $D G D_{F}=15.3 \mathrm{ps} ; 2^{\text {nd }}$ row: $\left.D G D_{F}=21.7 \mathrm{ps}\right)$. Initial signal SOP is aligned to one PSP. Worst case eye diagrams are shown on the right. 

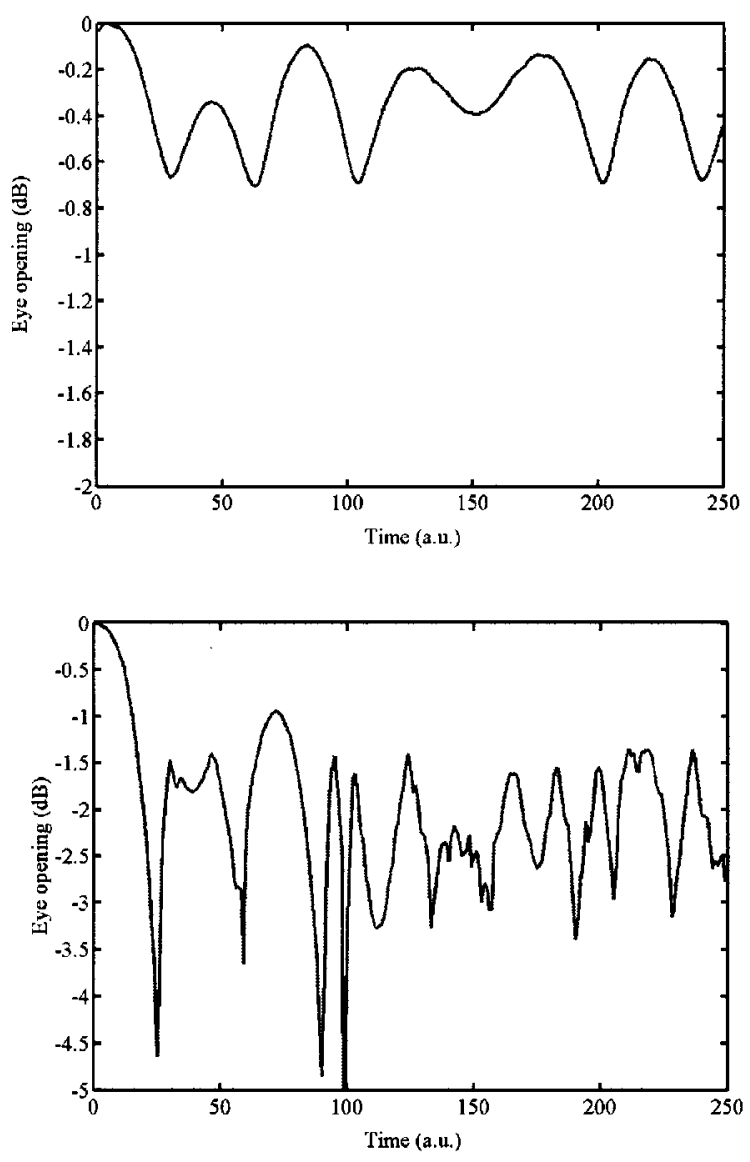

Figure 3 Eye closure evolution for a 1 -stage $\mathrm{PMDC}$ with $\mathrm{DGD}_{\mathrm{C}}=26$ ps. Same other parameters as in Fig. 2.(upper row: fibre $D G D_{F}=15.3$ ps; lower row: $D G D_{F}=21.7 \mathrm{ps}$ ).

Fig. 2 shows two evolution curves obtained for $D_{G D}=16$ ps for two fiber configurations and input SOP close to one PSP. In the upper row, we report the case when the fiber has $\mathrm{DGD}_{\mathrm{F}}=15.3 \mathrm{ps}$. Here, after the initial locking, the eye suddenly suffers from a significant penalty, meanwhile the PMDC switches to the minimisation mode, so that no other penalty is observed since then. Note that $\mathrm{DGD}_{\mathrm{F}}<\mathrm{DGD}_{\mathrm{C}}$, so the switching produces a significant penalty that was neither predicted by the $1^{\text {st }}$-order model presented heretofore.

On the right (same row), we show the eye diagram taken during this switching time, which indeed shows around $1.5 \mathrm{~dB}$ penalty. We note that, in the same fibre, an uncompensated system can suffer, in the worst case, from around 
the same penalty.

This result is suggesting possible limitations of the PMDCs. Still, the worst results are obtained when, due to the statistical variations, the fibre reaches a higher $\mathrm{DGD}_{\mathrm{F}}$ value, which is exactly the case when the PMDC is really needed. An apparent example is shown in the $2^{\text {nd }}$ row in Fig. 2: here $D G D_{F}=21.7$ ps and the PMDC does not reach a stable condition, so that the eye closure tends to oscillate repeatedly to unacceptable values $(\ll-3 \mathrm{~dB})$. An example of such high-penalty eye diagram is shown on the right. We outline that, again, this dynamics cannot be predicted by the usual quasi-static OP analysis. Indeed in this case, the quasi-static analyisis would show a very limited penalty, which cannot be attained in a dynamical environment.

Some of the previous results can be understood by means of the above firstorder model. According to that model, a PMDC with higher $\mathrm{DGD}_{\mathrm{C}}(\gg 16 \mathrm{ps})$ might be more useful in the dynamical regime (producing a $\vec{\Omega}$ vector always parallel to $\vec{s}$ ). To check this possibility, we run dynamical simulations for the same above cases, but now using $\mathrm{DGD}_{\mathrm{C}}=26 \mathrm{ps}$. Now we find that when the fibre $\mathrm{DGD}_{\mathrm{F}}$ is comparable to $T_{b i t}$, still the PMDC dynamics is strongly affected (see Fig. 3). This clearly indicates that for high values of $\mathrm{DGD}_{\mathrm{F}}$ and $\mathrm{DGD}_{\mathrm{C}}$ high-order PMD effects come into play. These effects cannot actually be neglected and again this eventually prevents the PMDC to attain a stable condition. This is clearly shown in both trends reported in Fig. 3 (for $\mathrm{DGD}_{\mathrm{F}}=15.3$ and 21.7 , respectively). Furthermore, in this case some oscillating behaviour is observed also for low $\mathrm{DGD}_{\mathrm{F}}$ (Fig. $31^{\text {st }}$ row). These results seem to indicate that, as was found for the quasi-static approach [4] [5], a quite high $\mathrm{DGD}_{\mathrm{C}}$ value can produce worse performance also in the dynamical regime.

\section{CONCLUSIONS}

A first-order model indicates that a single-stage PMDC with feedback may produce relevant system impairments if it locks to an unstable condition and if $\mathrm{DGD}_{\mathrm{F}} / \mathrm{DGD}_{\mathrm{C}}>1$. Numerical simulations confirmed that PMDCs could suffer from this effect. Despite the first order model, higher length PMDCs could suffer even more due to $2^{\text {nd }}$ order PMD.

These results might hold also for a single stage with variable length and for a double stage PMDC, but this issue is still being investigated. On the other hand, PMDCs using polarization scrambling [8] and PMDCs with no feedback (e.g., [9],[10]) could not suffer from these dynamical limitations.

The obtained results seriously indicate that the Outage Probability may not completely describe the performance of a PMDC: it provides indeed an upper bond, a bound that could not always be met. 
Finally, in our scheme we assumed an initial startup of the PMDC, with an exhaustive search. In a practical environment, the PMDC may lock to an unstable condition either because of noise fluctuations, or the random evolution of fibre parameters.

\section{ACKNOWLEDGMENTS}

The author acknowledges stimulating discussions with E. Forestieri and the fruitful cooperation with the Optical Networks Group of Marconi Communications in Genoa, whose activity has stimulated and supported this work. This work was supported by Marconi Communications, under a grant.

\section{REFERENCES}

[1] H. Sunnerud, M. Karlsson, C. Xie, P.A. Andrekson, "Polarization Mode Dispersion in High Speed Fiber-Optic Transmission Systems" J. Light. Technol., 20, 12 2204-2219 (2002).

[2] F. Curti, B. Daino, G. De Marchis, and F. Matera, "Statistical treatment of the evolution of the principal states of polarization in single-mode fibers," J. Lightwave Technol., vol. 8, pp. $1162-1166,1990$.

[3] H. Bulow, "System outage probability due to first and second order PMD" IEEE Photon. Technol. Lett. 10, 696-699 (1998)

[4] H. Sunnerud, C. Xie, M. Karlsson, R. Samuelsson, P.A. Andrekson, "A comparison between different PMD compensation techniques" J. Light. Technol., 20, 3, 368-378 (2002).

[5] C. Xie, and H. Haunstein, "Optimum Length of One-Stage Polarization-Mode Dispersion Compensators With a Fixed Delay Lin", " IEEE Photon. Technol. Lett. 15, 9, 1228-1231 (2003)

[6] A. Galtarossa, L. Palmieri, M. Schiano, T. Tambosso, "Single-End Polarization Mode Dispersion Measurement Using Backreflected Spectra Through a Linear Polarizer", J. Lightwave Technol., 17, 10, 1835-1842 (1999).

[7] R. M. A. Azzam N.M. Bashara, "Ellipsometry and polarized light", Elsevier Science Ed., Amsterdam, 1999.

[8] H. Y. Pua, K. Peddanarppagari, B. Zhu, C. Allen, K. Demarest and R. Hui, "An adaptive First order Polarization Mode Dispersion compensation system aided by polarization scrambling: theory and demonstration", J. Light. Technol. 18, 6, 832-841 (2000).

[9] P. Chou, J. M. Fini, H. Haus, "Demonstration of a feed-forward PMD compensation technique" IEEE Photon. technol. Lett. 14, 2, 161-163 (2002).

[10] J. M. Fini, P. Chou, H. Haus, "Estimate of polarization dispersion parameters for compensation with reduced feedback" IEEE Photon. Technol. Lett. 14, 2, 161-163 (2002). 